\title{
Erratum to: Rough Burgers-like equations with multiplicative noise
}

\author{
Martin Hairer • Hendrik Weber
}

Published online: 30 October 2013

(C) Springer-Verlag Berlin Heidelberg 2013

\section{Erratum to: Probab. Theory Relat. Fields (2013) 155:71-126 DOI 10.1007/s00440-011-0392-1}

Unfortunately, the proof of Proposition 4.8 in the original article is not correct. In fact, equation (4.60), where $R^{\theta}$ is rewritten as a stochastic integral, has no meaning because the integrand is not adapted. Hence in (4.61) we are not allowed to apply the Burkholder-Davies-Gundy inequality.

The argument was corrected in [1, Lemma 3.6 and Corollary 3.7] in a more complicated situation. In order to transfer these statement to the situation in the original article some small changes are needed. First of all, the regularity of the linearised process $X$ should be measured in a Hölder norm with slightly bigger index $\alpha_{\star}$ than the solution $u$. This can be done without further problems. With this change, the definition of the stopping time $\tau_{K}^{X}$ in (4.53) should be replaced by the following.

For $K>0$ and for an $\alpha_{\star} \in(\alpha, 1 / 2)$ we introduce the stopping time

$$
\tau_{K}^{X}=\inf \left\{t \in[0, T]: \sup _{\substack{x_{1} \neq x_{2} \\ 0 \leq s_{1}<s_{2} \leq t}} \frac{\left|X\left(s_{1}, x_{1}\right)-X\left(s_{2}, x_{2}\right)\right|}{\left|s_{1}-s_{2}\right|^{\alpha_{\star} / 2}+\left|x_{1}-x_{2}\right|^{\alpha_{\star}}}>K\right\}
$$

The online version of the original article can be found under doi:10.1007/s00440-011-0392-1.

M. Hairer $(\varangle) \cdot$ H. Weber

Mathematics Department, University of Warwick,

Coventry CV4 7AL, UK

e-mail: M.Hairer@Warwick.ac.uk

H. Weber

e-mail: hendrik.weber@warwick.ac.uk 
With this changed definition, Lemma 3.6 of [1] implies, in the notation of the original article, the following result.

Lemma 1 Suppose that $0<\alpha<\alpha_{\star}<\frac{1}{2}$ and let $\tau$ be a stopping time that almost surely satisfies

$$
0 \leq \tau \leq \tau_{K}^{X} \wedge T
$$

For every $0 \leq t \leq T$ we set

$$
\begin{aligned}
\tilde{\theta}(t) & :=\theta(t \wedge \tau), \\
\tilde{\Psi}^{\theta}(t) & :=\int_{0}^{t \wedge \tau} S(t-r) \tilde{\theta}(r) d W(r), \\
\tilde{X}(t) & :=\int_{0}^{t \wedge \tau} S(t-r) d W(r), \\
\tilde{R}^{\theta}(t ; x, y) & :=\delta \tilde{\Psi}^{\theta}(t ; x, y)-\tilde{\theta}(t, x) \delta \tilde{X}(t ; x, y) .
\end{aligned}
$$

Then, for any $p$ large enough and for any $\gamma>0$ such that

$$
\gamma<\alpha_{\star}+\alpha-\frac{1}{p}-\sqrt{\frac{1}{2 p}\left(1+\alpha-\alpha_{\star}\right)},
$$

the following bound holds true:

$$
\sup _{0<t \leq T} \mathbb{E}\left|\tilde{R}^{\theta}(t)\right|_{\Omega \mathcal{C} \gamma}^{p} \lesssim\|\theta\|_{p, \alpha}^{p}
$$

The statement given here is actually slightly stronger than the bound stated in [1] because the norm appearing on the left hand side of (2) is bounded uniformly in $t$ instead of allowing a blow up near 0 . In [1] we had to introduce this blowup due to a slightly modified definition of the Gaussian process $X$ : the process used in [1] does not start at 0 , but with stationary initial condition, which was convenient for other reasons. When going through the proof given in [1], one realises that when considering the process with zero initial condition, one can apply bound (3.74) for all times $t$ and there is no need to use (3.75) for small times.

Based on this version of Lemma 1, it is then straightforward to use the a priori information on the time regularity of $R^{\theta}$, combined with the fact that the "tilde" processes coincide with the "non-tilde" processes before time $\tau$, to obtain the bound

$$
\mathbb{E}\left[\left\|R^{\theta}\right\|_{C^{\kappa}\left([0, \tau] ; \Omega C^{2 \alpha}\right)}^{p}\right] \leq C(K, T)\|\theta\|_{p, \alpha}^{p},
$$

for sufficiently small values of $\kappa$ and sufficiently large values of $p$, as required. 
Acknowledgments The authors are very grateful to the anonymous referee of [1] for pointing out this mistake.

\section{Reference}

1. Hairer, M., Maas, J., Weber, H.: Approximating rough stochastic PDEs (arXiv e-prints) 